\title{
Acute pancreatitis induced by placement of a duodenal stent
}
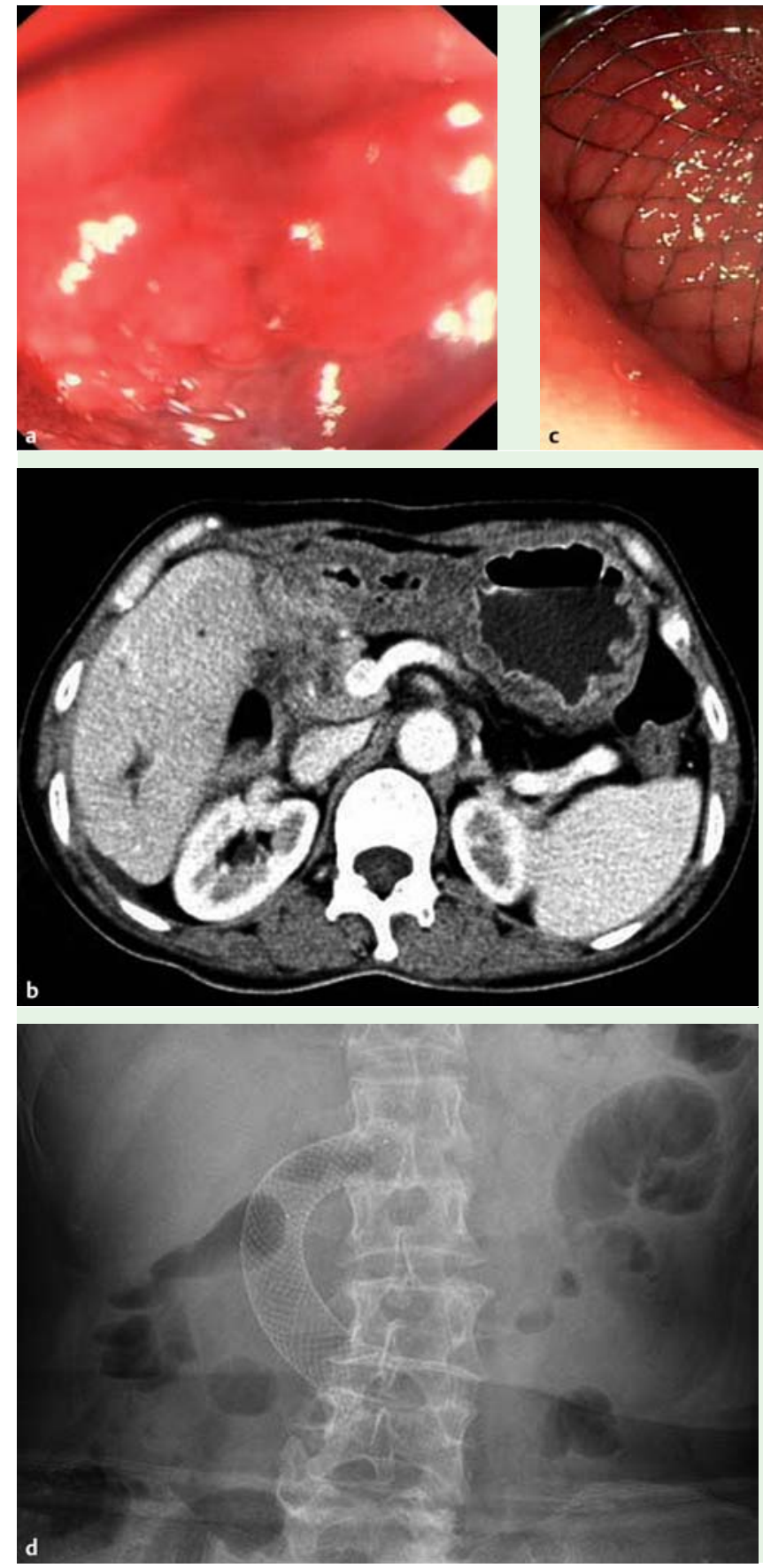

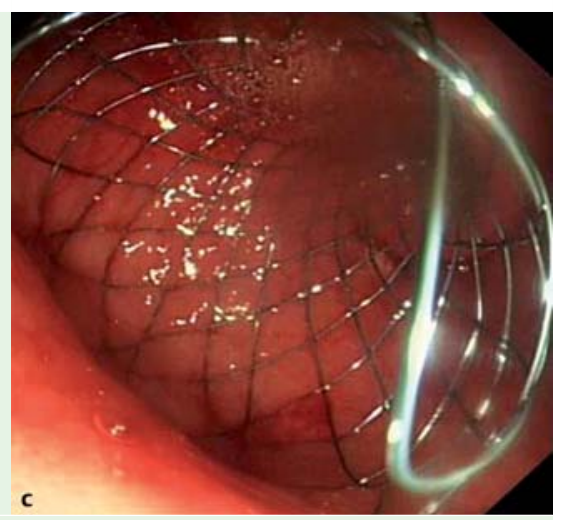

Fig. 1 Endoscopic and radiologic appearance of the patient with cholangiocellular carcinoma before and after placement of a duodenal stent. a Before stent placement, duodenal obstruction in the descending duodenum was found by a diagnostic gastroscopy. $\mathbf{b}$ Computed tomography scan showed that the cholangiocellular carcinoma was located in the porta hepatis area and left liver, infiltrating the duodenum and leading to luminal stenosis. c A second gastroscopy confirmed the correct position of the stent, the proximal part of which was in the gastric antrum. $\mathbf{d}$ A plain abdominal radiograph 1 day after stent placement also confirmed the correct position of the stent.
Placement of metal stents is an effective therapy for patients with duodenal obstruction. Compared with palliative surgical treatment, it yields higher clinical success rates and shorter hospital stays [1,2]. Acute pancreatitis following this therapy has been reported only rarely. We report a case of acute pancreatitis following placement of a duodenal metal stent.
A 59-year-old Chinese woman was admitted to our hospital on 2 April 2013 with a history of cholangiocellular carcinoma in the porta hepatis area. She had been suffering from aphagosis and vomiting for 2 weeks. The diagnostic gastroscopy revealed duodenal obstruction in the descending duodenum ( $\bullet$ Fig. 1 a). A computed tomography (CT) scan showed an obscure boundary mass in the porta hepatis area, infiltrating the duodenum ( $\bullet$ Fig. 1 b). Placement of a metal duodenal stent was performed successfully. After the endoscopic therapy, the patient kept complaining of upper abdominal pain. At first, this symptom was attributed to possible stent compression. One day later, the pain had not reduced and vomiting re-started. A second gastroscopy $(\bullet$ Fig. 1 c) and a plain abdominal radiograph ( $\bullet$ Fig.1d) showed that the stent had not moved. Meanwhile, blood tests showed an elevated amylase of $1010 \mathrm{U} / \mathrm{L}$, which strongly indicated the development of acute pancreatitis.

After 3 days of therapy involving absolute zero diet (i.e. no food or drink permitted), omeprazole, octreotide, and fluid infusion, the patient's symptoms of upper abdominal pain and vomiting resolved. The patient was thus diagnosed with acute pancreatitis caused by duodenal stent placement.

Compression of the duodenal papilla by the stent can lead to increased pressure in the pancreatic duct, which results in acute pancreatitis. A stent bridging the duodenal papilla is a significant predictor for the development of acute pancreatitis [3]. Due to the lack of specific symptoms or the inexperience of gastrointestinal physicians, acute pancreatitis following duodenal stent placement is easy to misdiagnose.

Endoscopy_UCTN_Code_CPL_1AH_2AD

Competing interests: None

\section{C. Lou', J. M. Yang ${ }^{1}$, J. J. Ke ${ }^{1}$, W. Q. Wu ${ }^{1}$, B. Zhou ${ }^{2}$}

${ }^{1}$ Department of Gastroenterology, Zhejiang Provincial People's Hospital, Hangzhou, China

${ }^{2}$ Key Laboratory of Nutrition and Metabolism, Institute for Nutritional Sciences, Shanghai Institutes for Biological Sciences, Graduate School of the Chinese Academy of Sciences, Chinese Academy of Sciences, Shanghai, China

\section{References}

1 van Hooft JE, Uitdehaag MJ, Bruno MJ et al. Efficacy and safety of the new WallFlex enteral stent in palliative treatment of malignant gastric outlet obstruction (DUOFLEX study): a prospective multicenter study. Gastrointest Endosc 2009; 69: 1059-1066

2 Graber I, Dumas R, Filoche B et al. The efficacy and safety of duodenal stenting: a pro- 
spective multicenter study. Endoscopy 2007; 39: 784-787

3 Shi-Yi L, Ai-Wu M, Yi-Ping J et al. Placement of duodenal stents across the duodenal papilla may predispose to acute pancreatitis: a retrospective analysis. Diagn Interv Radiol 2012; 18: 360 - 364
Bibliography

DOI http://dx.doi.org/

10.1055/s-0033-1344423

Endoscopy 2013; 45: E256-E257

(c) Georg Thieme Verlag KG

Stuttgart · New York

ISSN 0013-726X

\section{Corresponding author}

W. Q. Wu, MD

Department of Gastroenterology

Zhejiang Provincial People's Hospital

Hangzhou 310014

China

Fax: +86-571-85131448

48634474@qq.com 